\title{
ESTIMATION OF ANGLE ELEMENTS OF EXTERIOR ORIENTATION FOR UAV IMAGES BASED ON INS DATA AND AERIAL TRIANGULATION PROCESSING
}

\author{
Damian Wierzbicki \\ Military University of Technology, Poland \\ damian.wierzbicki@wat.edu.pl
}

\begin{abstract}
So far, there are too many examinations concerning the possibility of the application UAV in photogrammetric studies. Unmanned aerial vehicles are usually equipped with low cost and low grade Inertial Navigation System (INS). In relation to determination the value of the angle orientations of the camera on low cost UAV mainly inertial sensors based on micro-electromechanical systems are being used (MEMS). In this case, the typical accuracy of determining the angles of the platform can reach up to 0.5 degree. In the paper, results of comparison of the angle elements of exterior orientation between the transformation model from the INS sensor to camera sensor, and the aerial triangulation method are presented. Two research methods were used in this paper, i.e. the method of transformation between the frame of the INS sensor and the frame of the camera sensor and the method of digital aerial triangulation for determination of the angle. The research experiment was realized for 789 images data from Trimble UX-5 sensor. The flight mission was conducted above Liwiec river in 2015. The results of the comparison show that the mean difference of the angle elements of exterior orientation between both methods amounts to $\pm 1.5^{\circ}$.
\end{abstract}

Keywords: photogrammetry, INS, UAV, aerial triangulation, exterior orientation optimisation.

\section{Introduction}

The sensor INS (Inertial Navigation System) is the basic sensor used to define parameters of the orientation of an unmanned platform in space. Apparatus of the INS device has built in components of gyroscopes for determination of the angles of the sense of direction and accelerometers for determination of acceleration of the platform [1]. The gyroscopes enable the reconstruction of the angle elements of YPR orientation (Yaw, Pitch, Roll) in 3 axis for an unnamed moving platform.

The occurrence of errors in gyroscope and accelerometers measurements has big impact on accuracy of determination of the angle YPR elements for unmanned platforms [2]. Moreover, action of external factors (like the speed of wind or atmospheric fallout) is essential to determine the precise values of the YPR angles of rotation [3]. Determination of the angle YPR elements has huge meaning for the use in air photogrammetry. The YPR parameters allow to determinate approximate values of the angle elements of exterior orientation in the area of aviation. Values of the angle of exterior orientation are determined with use of OPK parameters (Omega, Phi, Kappa) in relation to the camera sensor placed in the unmanned platform [4].

The purpose of the work was to determine the values of OPK angles based on two research methods. The first research method was based on the transformation YPR angle from the INS sensor for reconstructing the angle OPK elements for the camera sensor. The second method assumed the determination of angular values of the OPK based on the aerial triangulation. The work compares the OPK angle values based on the applied research methods. An analysis of the accuracy of the obtained results was also carried out. The calculations were carried out for the navigation data obtained from the unmanned Trimble UX-5 platform.

The article was divided into 5 sections and references were added at the end of this paper.

\section{Mathematical model for designation the angle elements of exterior orientation}

Values of angle elements of exterior orientation are determined on the basis of transformation from the frame of the INS sensor to the frame of a photogrammetric camera sensor as below [5]:

$$
C_{E}^{B}=T_{b}^{B} \cdot\left(C_{n 0}^{n^{\prime}} \cdot C_{e}^{n 0} \cdot\left(C_{e}^{n i}\right)^{T} \cdot C_{b}^{n i}\right)^{T} \cdot\left(T_{n}^{E}\right)^{T},
$$

where $C_{E}^{B}$ - orthogonal matrix, includes OPK angle elements; 
$C_{E}^{B}=\left[\begin{array}{ccc}\cos \varphi \cos \kappa & -\cos \varphi \sin \kappa & \sin \varphi \\ \cos \varpi \sin \kappa+\sin \varpi \sin \varphi \cos \kappa & \cos \varpi \cos \kappa-\sin \varpi \sin \varphi \sin \kappa & -\sin \varpi \cos \varphi \\ \sin \varpi \sin \kappa-\cos \varpi \sin \varphi \cos \kappa & \sin \varpi \cos \kappa+\cos \varpi \sin \varphi \sin \kappa & \cos \varpi \cos \varphi\end{array}\right] ;$

$\varphi-$ Phi angle,,$\varphi=\arcsin C_{E}^{B}(1,3)$;

$\varpi$ - Omega angle, $\varpi=-\operatorname{arctg}\left(\frac{C_{E}^{B}(2,3)}{C_{E}^{B}(3,3)}\right)$;

$\kappa-$ Kappa angle, $\kappa=-\operatorname{arctg}\left(\frac{C_{E}^{B}(1,2)}{C_{E}^{B}(1,1)}\right)$;

$T_{b}^{B}=\left[\begin{array}{ccc}-1 & 0 & 0 \\ 0 & 1 & 0 \\ 0 & 0 & -1\end{array}\right], T_{b}^{B}-$ matrix determinant equals $1 ;$

$C_{n 0}^{n^{\prime}}=\left[\begin{array}{ccc}1 & 0 & 0 \\ 0 & 1 & 0 \\ 0 & 0 & 1\end{array}\right], C_{n 0}^{n^{\prime}}-$ singular matrix

$C_{e}^{n 0}=\left[\begin{array}{ccc}-\sin B_{0} \cdot \cos L_{0} & -\sin B_{0} \cdot \sin L_{0} & \cos B_{0} \\ -\sin L_{0} & \cos L_{0} & 0 \\ -\cos B_{0} & -\cos B_{0} \cdot \sin L_{0} & -\sin B_{0}\end{array}\right]$,

$C_{e}^{n 0}=f\left(B_{0}, L_{0}\right)$, matrix includes unmanned aircraft coordinates in ellipsoidal frame,

$\left(B_{0}, L_{0}\right)$ - unmanned aircraft coordinates refer to the central point of the flight trajectory,

$C_{e}^{n i}=\left[\begin{array}{ccc}-\sin B_{i} \cdot \cos L_{i} & -\sin B_{i} \cdot \sin L_{i} & \cos B_{i} \\ -\sin L_{i} & \cos L_{i} & 0 \\ -\cos B_{i} & -\cos B_{i} \cdot \sin L_{i} & -\sin B_{i}\end{array}\right]$,

$C_{e}^{n i}=f\left(B_{i}, L_{i}\right)$, matrix includes unmanned aircraft coordinates in ellipsoidal frame;

$\left(B_{i}, L_{i}\right)$ - unnamed aircraft coordinates for each measuring epoch;

$C_{b}^{n i}$ - orthogonal matrix, includes YPR angle elements,

$C_{b}^{n i}=\left[\begin{array}{ccc}\cos \psi \cdot \cos \theta & \cos \psi \cdot \sin \theta \cdot \sin \phi-\sin \psi \cdot \cos \phi & \cos \psi \cdot \sin \theta \cdot \cos \phi+\sin \psi \cdot \sin \phi \\ \sin \psi \cdot \cos \theta & \sin \psi \cdot \sin \theta \cdot \sin \phi+\cos \psi \cdot \cos \phi & \sin \psi \cdot \sin \theta \cdot \cos \phi-\cos \psi \cdot \sin \phi \\ -\sin \theta & \cos \theta \cdot \sin \phi & \cos \theta \cdot \cos \phi\end{array}\right] ;$

$\phi-$ Roll angle;

$\theta-$ Pitch angle;

$\psi-$ Yaw angle;

$T_{n}^{E}=\left[\begin{array}{ccc}0 & 1 & 0 \\ 1 & 0 & 0 \\ 0 & 0 & -1\end{array}\right], T_{n}^{E}-$ matrix determinant equals 1.

Accuracy of angle elements of exterior orientation is between $1^{\circ}$ and $5^{\circ}$ based on equation (1) [6]. Values of OPK angle are determined in the sequential procedure based on equation (1) for each measuring epoch. Values of angle elements of external orientation obtained from equation (1) require additional correction in order to increase the accuracy and precision. Figure 1. shows dependencies between coordinate systems in navigation and photogrammetry. 


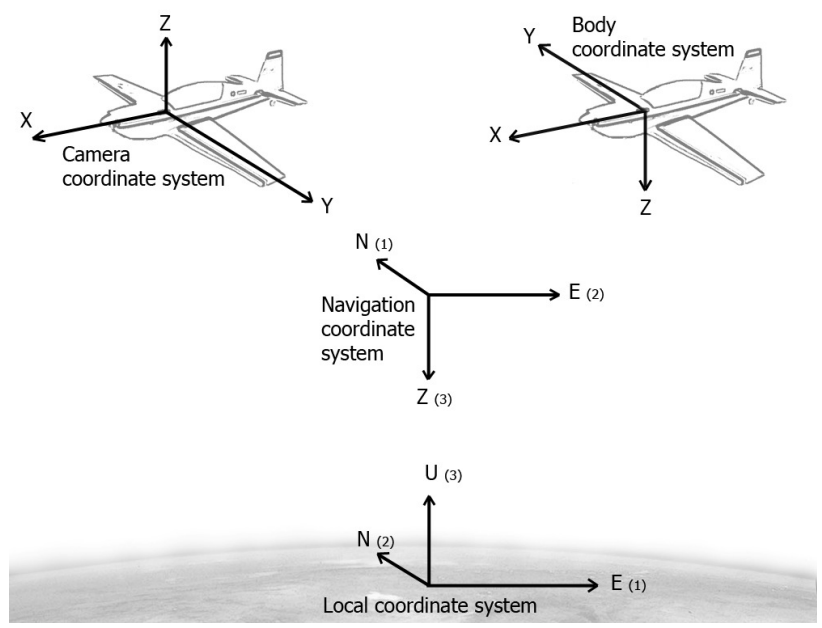

Fig. 1. Coordinate systems used in navigation and photogrammetry [7]

In the case of photogrammetry high accuracy and precision of OPK elements is possible by using the digital aerial triangulation process. Within the framework of the digital aerial triangulation model accuracy of determination of the angle OPK elements is higher than $0.1^{\circ}$. Equation based on the digital aerial triangulation model can be expressed as below [8,9]:

$$
\left\{\begin{array}{l}
x_{k}-x_{0}=-f_{k}\left[\frac{r_{11}\left(X_{T}-X_{k r}\right)+r_{21}\left(Y_{T}-Y_{k r}\right)+r_{31}\left(Z_{T}-Z_{k r}\right)}{r_{13}\left(X_{T}-X_{k r}\right)+r_{23}\left(Y_{T}-Y_{k r}\right)+r_{33}\left(Z_{T}-Z_{k r}\right)}\right] \\
y_{k}-y_{0}=-f_{k}\left[\frac{r_{12}\left(X_{T}-X_{k r}\right)+r_{22}\left(Y_{T}-Y_{k r}\right)+r_{32}\left(Z_{T}-Z_{k r}\right)}{r_{13}\left(X_{T}-X_{k r}\right)+r_{23}\left(Y_{T}-Y_{k r}\right)+r_{33}\left(Z_{T}-Z_{k r}\right)}\right],
\end{array}\right.
$$

where $\left(x_{k}, y_{k}\right)$ - image coordinates;

$\left(x_{0}, y_{0}\right)$ - center point of image coordinates;

$f_{k}$ - focal length;

$\left(X_{T}, Y_{T}, Z_{T}\right)$-coordinates of ground control points in terrain frame;

$\left(X_{k r}, Y_{k r}, Z_{k r}\right)$-coordinates of the projection center, expressed in terrain frame;

$R=\left[\begin{array}{lll}r_{11} & r_{21} & r_{31} \\ r_{12} & r_{22} & r_{32} \\ r_{13} & r_{23} & r_{33}\end{array}\right] ;$

$R$ - orthogonal matrix, the matrix contains angle elements of exterior orientation;

$R=R(\omega, \varphi, \kappa)$

$(\omega, \varphi, \kappa)$ - angle elements of external orientation.

Based on equation (2) the angle values $(\omega, \varphi, \kappa)$ are determined using the least squares methods in the iterative process. Angle parameters $(\omega, \varphi, \kappa)$ are related to each image in the particular profile. Technique of determination of elements of exterior orientation for the whole image block is usually based on the method of independent bundles.

\section{The research experiment}

Within the framework of the research experiment comparison of the angle elements of exterior orientation between the transformation model from the INS sensor to the camera sensor and the aerial triangulation method was conducted. Input parameters of the angles of rotation YPR for transformation process come from the data of gyroscopes placed on the unmanned platform Trimble UX-5. Ellipsoidal coordinates of the unnamed aircraft were registered by the GPS sensor placed on the Trimble UX-5 platform. Expediently the parameters of the position and orientation of the Trimble UX-5 sensor are recordable in the universal text format "log" for each image taken during the photogrammetric flight $[10 ; 11]$. Trimble UX-5 platform took 789 images in JPEG format with use of 
Sony NEX-5R digital camera during the photogrammetric flight. All images located in 40 profiles were taken from the altitude about $100 \mathrm{~m}$ (see Fig. 2). The flight mission was conducted in the east west direction, on the assumption that crosswise and oblong cove equals $75 \%$. The test area was placed in Starowola city by the Liwiec river near Warsaw conducted in good weather conditions and the wind speed no more than $2 \mathrm{~m} \cdot \mathrm{s}^{-1}$.

To needs of the digital aerial triangulation process 14 ground control points were placed and signalized on the test area, which were measured using the satellite technology GPS RTK. The accuracy of determination of the ground control points coordinates equal about $0.03 \mathrm{~m}$. The digital aerial triangulation process was conducted in UASMaster software based on the algorithm of the compensation with the method of bundle adjustment [12;13]. Obtained dimension of the pixel in the digital aerial triangulation equals $0.04 \mathrm{~m}$. Destination coordinate frame in the digital aerial triangulation process was set to PUWG 2000 zone 7.
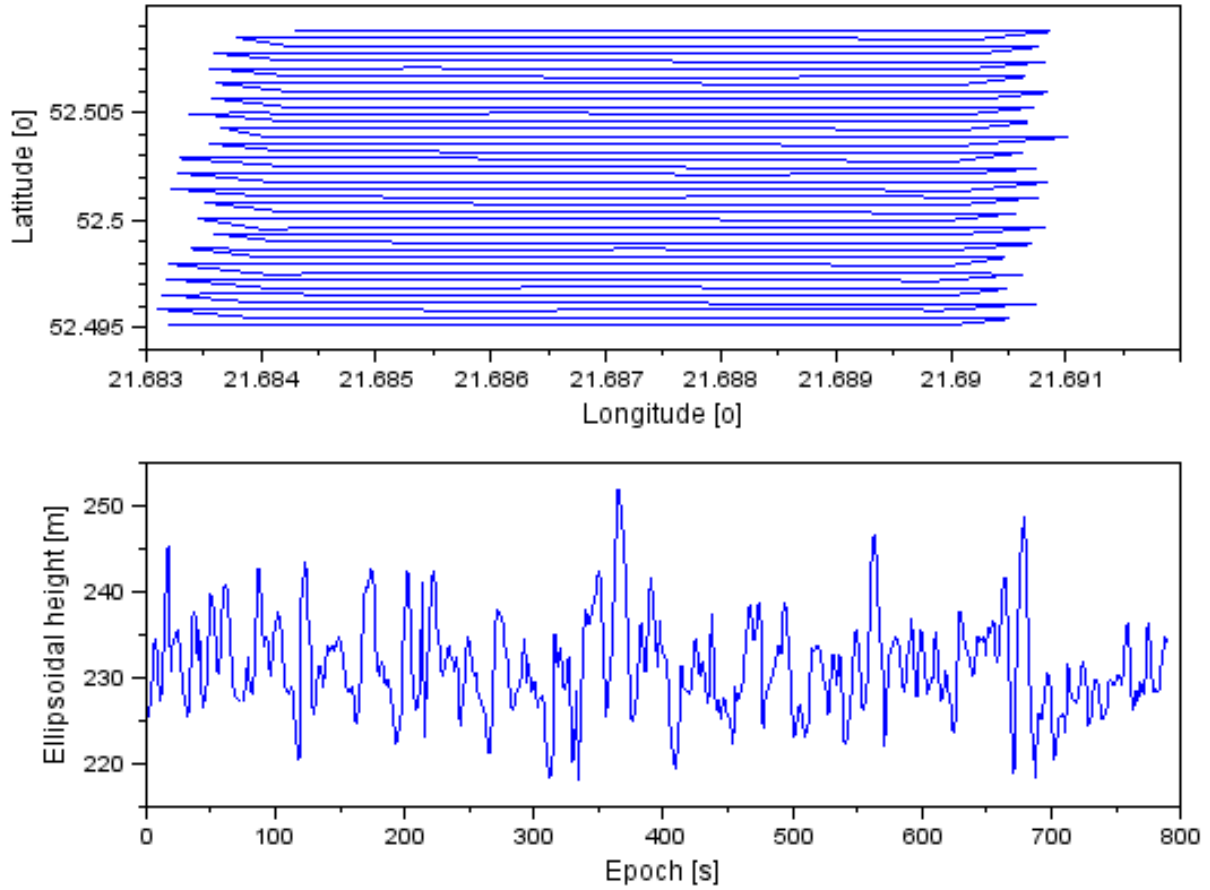

Fig. 2. UAV trajectory in horizontal and vertical plane

\section{The results and discussion}

Comparison of the results obtained on the basis of transformation of the INS sensor to the digital camera and the digital aerial triangulation method was made in the second chapter of the article. Value of the difference for $(\omega, \varphi, \kappa)$ parameters between the results from both research methods are determined as below:

$$
\left\{\begin{array}{l}
d \omega=\omega_{\text {TRANSF }}-\omega_{A E R O} \\
d \varphi=\varphi_{\text {TRANSF }}-\varphi_{A E R O} \\
d \kappa=\kappa_{\text {TRANSF }}-\kappa_{\text {AERO }}
\end{array},\right.
$$

where $\left(\omega_{\text {TRANSF }}, \varphi_{\text {TRANSF }}, \kappa_{\text {TRANSF }}\right)$ - values of angle elements $(\omega, \varphi, \kappa)$ based on dependence from equation (1);

$\left(\omega_{A E R O}, \varphi_{A E R O}, \kappa_{\text {AERO }}\right)$ - values of angle elements $(\omega, \varphi, \kappa)$ based on equation (2).

Fig. 3 presents the values of comparison of $\omega$ angle based on the method from equation (1) and equation (2). Average difference for $\omega$ angle value equals $-1.09^{\circ}$ with standard deviation $5.37^{\circ}$. The amplitude of the obtained results for $d \omega$ parameter equals between $-25.10^{\circ}$ and $15.66^{\circ}$. Moreover, median value of $d \omega$ parameter equals $-0.97^{\circ}$. It should be noted that $68 \%$ of the obtained results for 
$d \omega$ parameter are between $-5^{\circ}$ and $5^{\circ}$. Next, over $92 \%$ of the results obtained for $d \omega$ parameter are between $-10^{\circ}$ and $10^{\circ}$.

Fig. 4 presents the values of comparison of $\varphi$ angle based on the method from equation (1) and equation (2). Average difference for $\varphi$ angle value equals $1.22^{\circ}$ with standard deviation $5.79^{\circ}$. The amplitude of the obtained results for $d \varphi$ parameter equals between $-19.45^{\circ}$ and $20.76^{\circ}$. Moreover, median value of $d \varphi$ parameter equals $1.25^{\circ}$. It should be noted that about $61 \%$ of the obtained results for $d \varphi$ parameter are between $-5^{\circ}$ and $5^{\circ}$. Next, about $91 \%$ of the results for $d \varphi$ parameter are between $-10^{\circ}$ and $10^{\circ}$.

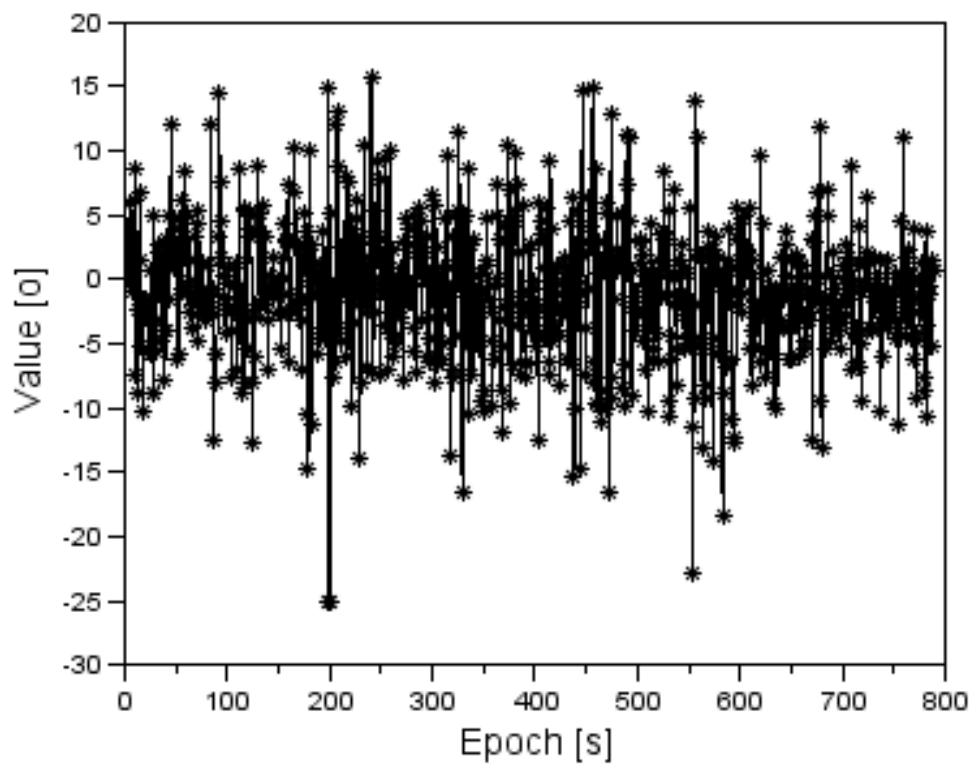

Fig. 3. Values of $d \omega$ parameter

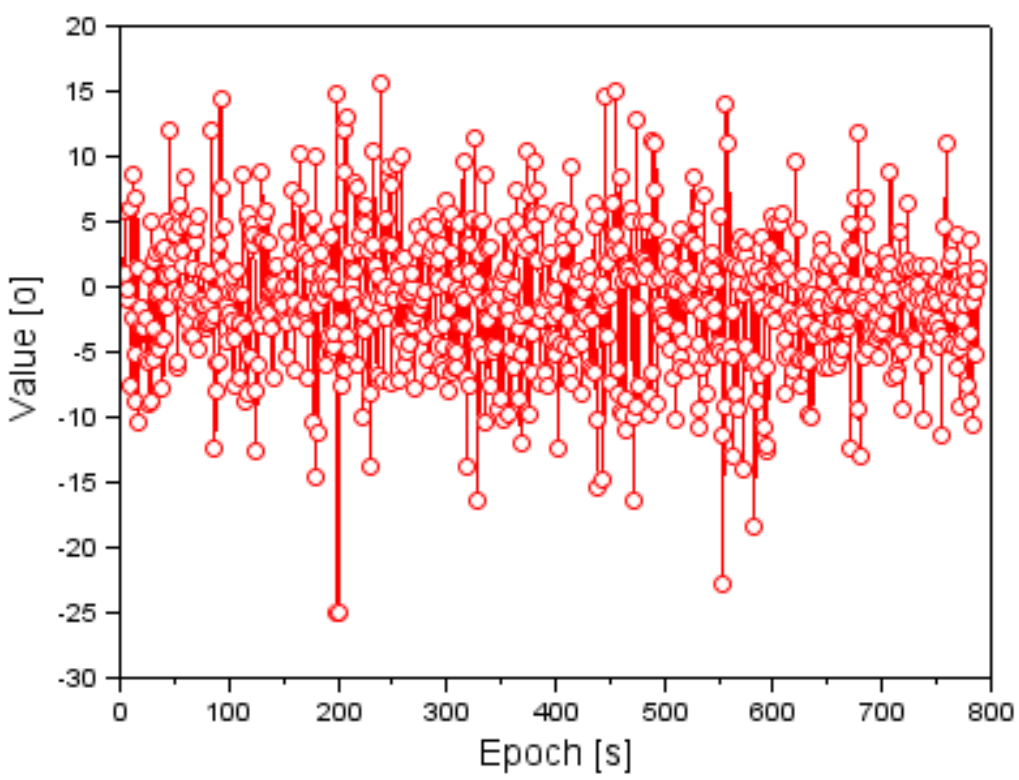

Fig. 4. Values of $d \varphi$ parameter

Fig. 5 presents the values of comparison of $\kappa$ angle based on the method from equation (1) and equation (2). Average difference for $\kappa$ angle value equals $0.87^{\circ}$ with standard deviation $9.68^{\circ}$. The amplitude of the obtained results for $d \kappa$ parameter equals between $-18.27^{\circ}$ and $25.63^{\circ}$. Moreover, median value of $d \kappa$ parameter equals $-1.47^{\circ}$. It should be noted that only $28 \%$ of obtained results for $d \kappa$ parameter are between $-5^{\circ}$ and $5^{\circ}$. Next, about $65 \%$ results for $d \kappa$ parameter are between $-10^{\circ}$ and $10^{\circ}$. Additionally $90 \%$ of the results for $d \kappa$ parameter are between $-15^{\circ}$ and $15^{\circ}$. 


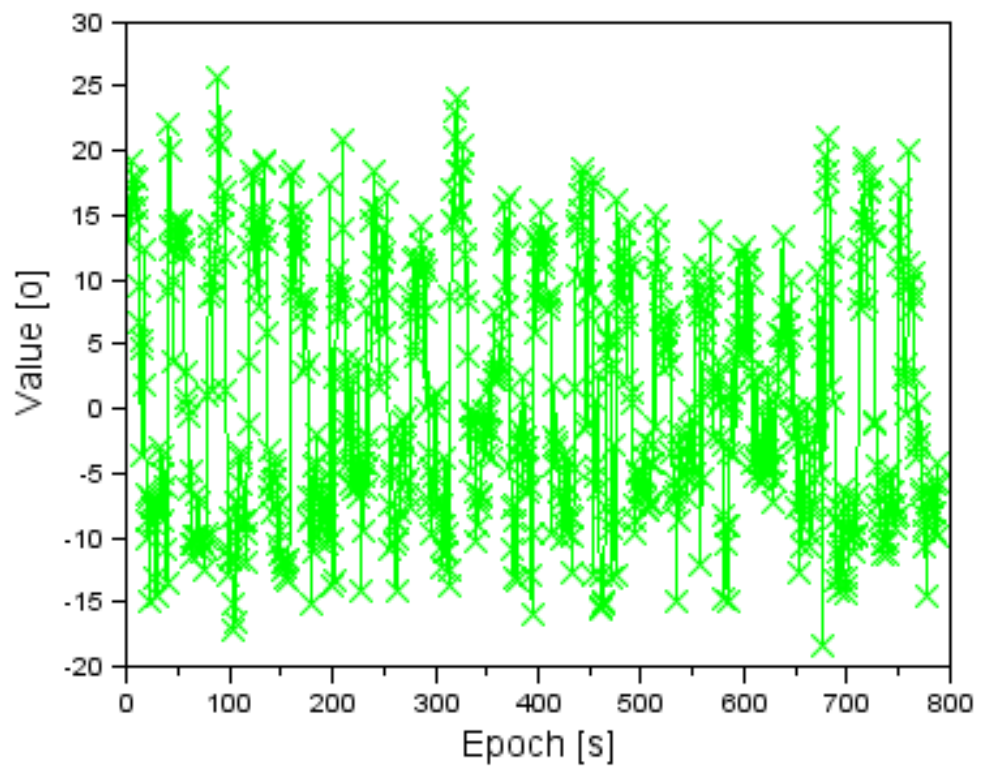

Fig. 5. Values of $d \kappa$ parameter

Table 1

Statistical analysis of Omega, Phi, Kappa angles

\begin{tabular}{|c|c|c|c|}
\hline \multirow{2}{*}{ Statistical size } & \multicolumn{3}{|c|}{ Angle, degrees } \\
\cline { 2 - 4 } & Omega & Phi & Kappa \\
\hline Average difference & -1.09 & 1.22 & 0.87 \\
\hline Amplitude of the results & 25.10 and 15.66 & -19.45 and 20.76 & -18.27 and 25.63 \\
\hline
\end{tabular}

Table 1 presents summary table values obtained for the statistical parameters Omega, Phi and Kappa angles. Based on the analyzes performed, it was found that the observations recorded by the INS sensor are possible to reconstruct the OPK angles with an accuracy of $1^{\circ}$. The aerial triangulation method increases the accuracy to $0.1^{\circ}$. Similar results were obtained in other works, where the average error for the roll, pitch and heading angles was $0.36^{\circ}$ [14]. Taking into account additional GPS observations, the accuracy for the yaw angle was even $0.20^{\circ}$ [15]. It has also been proved that the INS angle recording method can be used at the initial stage of the development of images obtained from a low altitude in order to determine approximate angular elements of the external orientation. The aerial triangulation method is more accurate; however, it requires more time to implement the research task.

\section{Conclusions}

In the article, the results of the research relating to determination the angle elements of exterior orientation by using UAV data were presented. Two research methods were used in this paper, i.e. the method of transformation between the frame of the INS sensor and the frame of the camera sensor and the method of digital aerial triangulation for determination of $(\omega, \varphi, \kappa)$ angle. The source data in the research test came from the photogrammetric experiment, which was conducted by the Liwiec river in 2015. Within the framework of the photogrammetric flight 789 images were taken using the Trimble UX-5 platform. Based on the conducted research tests and performed calculations the following conclusions were expressed:

- average difference of $(\omega, \varphi, \kappa)$ angle values equals: $-1.09^{\circ}$ for Omega angle, $1.22^{\circ}$ for Phi angle, $0.87^{\circ}$ for Kappa angle accordingly;

- amplitude of the obtained results for $(d \omega, d \varphi, d \kappa)$ parameters equals between $-25.10^{\circ}$ and $25.63^{\circ}$;

- standard deviation for $(d \omega, d \varphi, d \kappa)$ parameters equals about $6^{\circ}$ for Omega and Phi angle elements and about $10^{\circ}$ for Kappa angle. The results of the comparison show that the mean difference of the angle elements of exterior orientation between both methods amounts to $\pm 1.5^{\circ}$. 


\section{Acknowledgements}

Many thanks to Prof. Michal Kedzierski for his great help in acquiring data.

\section{References}

[1] Jafernik H., et al. . Assessment of suitability of radionavigation devices used in air, Scientific Journal of Silesian University of Technology. Series Transport, 90, pp. 99-112. ISSN: 0209-3324, 2016 DOI: $10.20858 /$ sjsutst.2016.90.9.

[2] Kolecki J. Determination of approximated exterior orientantion parameteres of terrestrial images using MEMS IMU, Archiwum Fotogrametrii, Kartografii i Teledetekcji, 24, pp.. 133-143, 2012 (In Polish).

[3] Kedzierski M., Fryskowska A., Wierzbicki D., Nerc P. Chosen aspects of the production of the basic map using UAV imagery Int. Arch. Photogramm. Remote Sens. Spatial Inf. Sci., XLI-B1, pp. 873-877, doi:10.5194/isprs-archives-XLI-B1-873-2016, 2016.

[4] Burman H. Calibration and orientation of airborne image and laser scanner data using GPS and INS, Ph D. thesis, ISBN 91-7170-565-1, Royal Institute of Technology, Sweden, 2000.

[5] Bürker M., Heimes F. J. New Calibration and Computing Method for Direct Georeferencing of Image and Scanner Data Using the Position and Angular Data of an Hybrid Inertial Navigation System, “OEEPE-Workshop Integrated Sensor Orientation", 17.9.-18.9.2001, Hannover, Germany, 2001.

[6] Eling C., Wieland M., Hess C., Klingbeil L., Kuhlmann H. Development and evaluation of a UAV based mapping system for remote sensing and surveying applications, The International Archives of the Photogrammetry, Remote Sensing and Spatial Information Sciences, Volume XL1/W4, International Conference on Unmanned Aerial Vehicles in Geomatics, 30 Aug-02 Sep 2015, Toronto, Canada, 2015.

[7] Khoshelham K., Gorte B. G. H. Aerial data acquisition for disaster management : exploiting the potential of integrated sensor orientation. In B. v.d. Walle, \& E. al. (Eds.), Information Systems for Crisis Response and Management : Proceedings of the Joint Third ISCRAM-CHINA and Gi4DM Conference, Harbin, China, 4-6 August 2008.

[8] Yi Z., Jiang G., Yanan Di. Speed-up matching method with navigation data for UAV remote sensing images of coastal region. "Remote Sensing of the Environment: 19th National Symposium on Remote Sensing of China". International Society for Optics and Photonics, 2015.

[9] Wierzbicki D. Determination of Shift/Bias in Digital Aerial Triangulation of UAV Imagery Sequences IOP Conf. Series: Earth and Environmental Science 95 (2017) 032033 World Multidisciplinary Earth Sciences Symposium (WMESS 2017) doi:10.1088/17551315/95/3/032033

[10] Wierzbicki D. , The prediction of position and orientation parameters of UAV for video imaging The International Archives of the Photogrammetry, Remote Sensing and Spatial Information Sciences, Volume XLII-2/W6, 2017 International Conference on Unmanned Aerial Vehicles in Geomatics, 4-7 September 2017, Bonn, Germany, 2017 doi:10.5194/isprs-archives-XLII-2-W6407-2017, 2017.

[11] Kedzierski M., Fryskowska A., Wierzbicki D., Grochala A., Nerc P.. Detection of Gross Errors in the Elements of Exterior Orientation of Low-Cost UAV Images, "2016 Baltic Geodetic Congress Gdansk", 2016, pp. 95-100, doi:10.1109/BGC.Geomatics.2016.26.

[12] Aguilar F. J., Fernández I., Casanova J. A., Ramos F. J., Aguilar M. A., Blanco J. L., Moreno J. C. 3D Coastal Monitoring from very dense UAV-Based Photogrammetric Point Clouds. In Advances on Mechanics, Design Engineering and Manufacturing (pp. 879-887). Springer, Cham, 2017.

[13] Mancini F. Dubbini M. Gattelli M. Stecchi F. Fabbri S. and Gabbianelli G. Using unmanned aerial vehicles (UAV) for high-resolution reconstruction of topography: the structure from motion approach on coastal environments. Remote Sensing, 5, pp.6880-6898, 2013.

[14] Kai-Wei C., Tsai M.L., Chu C.H. The development of an UAV borne direct georeferenced photogrammetric platform for ground control point free applications.Sensors 12.7: 9161-9180, 2012.

[15]Eling C., Klingbeil L., Kuhlmann H. Real-time single-frequency GPS/MEMS-IMU attitude determination of lightweight UAVs. Sensors, 15(10), 26212-26235, 2015. 